\title{
A Practical Hybrid-ARQ Protocol Using Noncoherent Orthogonal Modulation
}

\author{
Syed Amaar Ahmad and Matthew C. Valenti \\ Lane Dept. of Comp. Sci. and Elect. Eng. \\ West Virginia University \\ Morgantown, WV 26506-6109 \\ Email: asyed@mix.wvu.edu,mvalenti@wvu.edu
}

\begin{abstract}
This paper considers hybrid-ARQ under the constraints of noncoherent orthogonal modulation and hard-decision decoding. Information theoretic bounds on the throughput for such systems are derived. A practical coding technique based on Reed-Solomon codes is proposed and analyzed. The proposed hybrid-ARQ protocol is modified to allow relay nodes to service retransmission requests. The proposed protocol is suitable for IEEE 802.15.4/Zigbee networks using commodity off-the-shelf hardware.
\end{abstract}

\section{INTRODUCTION}

In slow (non-ergodic) fading channels, the instantaneous channel capacity varies from one transmitted codeword to the next [1]. If the code rate selected for a particular codeword is above the instantaneous capacity, then an information outage will occur. One way to reduce the occurrence of outages is to use adaptive modulation and coding, which could be used to adapt the rate to match the instantaneous capacity. However, such an approach requires transmitter channel state information (CSI) in the form of knowledge of the channel's signal to noise ratio (SNR).

A practical alternative to adaptive modulation and coding is hybrid-ARQ [2] that can mitigate fading effects. With hybrid-ARQ, the message is first encoded into a very low rate "mother" code. The codeword is then broken into several disjoint blocks. Blocks are transmitted one by one until the receiver is able to successfully decode and send back a positive acknowledgement (ACK). The information theory of hybridARQ in block fading is discussed in [3], with an emphasis on throughput and delay. With hybrid-ARQ, each time a new block is sent, the code rate is effectively lowered. Thus, this approach can be viewed as a form of adaptive rate coding. With hybrid-ARQ, the required transmit CSI is entirely embodied in the ACK messages. Even when large blocks are used, then the amount of CSI (in the form of ACK messages) does not need to be high.

The original information theoretic analysis of hybrid-ARQ presented in [2] is based on the assumption of Gaussian input symbols. While such an assumption is convenient because it allows the instantaneous capacity of each block to be expressed in the form $\log (1+S N R)$, it is unrealistic because practical modulation alphabets do not produce Gaussian symbols. More realistic bounds on throughput can be obtained by constraining the instantaneous capacity depending on the choice of modu- lation. In [4], we discuss the impact of modulation constraints upon the throughput of hybrid-ARQ from an informationtheoretic perspective. As a specific example, bounds on the throughput of hybrid-ARQ with QPSK and 16-QAM were calculated and compared against the throughput of an actual turbo coded hybrid-ARQ system that uses these modulations (from the UMTS-HSDPA standard).

A natural application of hybrid-ARQ is low cost wireless networks, such as personal area networks that follow the Bluetooth standard or sensor networks that follow the IEEE 802.15.4/Zigbee standard [5]. Such networks tend to use modulation techniques that can be noncoherently detected. For instance, Bluetooth uses nonorthogonal binary Gaussian frequency shift keying (GFSK) while Zigbee uses 16-ary pseudoorthogonal modulation. The information theoretic bounds on the performance of hybrid-ARQ applied to these two systems could be determined by applying the approach set forth in [4] and conditioning the instantaneous capacity on both the modulation and the noncoherent detector.

If one had access to the matched filter outputs on board the receiver, one could implement soft-decision decoding. If combined with a capacity approaching code, the system could be designed to closely approach the modulation-constrained information theoretic limits on throughput. However, most practical low cost wireless networks do not use soft-decision decoding. If a code is used at all, it is hard-decision decoded. This is a disadvantage from a performance standpoint, because hard-decision decoding always performs worse than softdecision decoding. However, the use of hard-decisions makes for a convenient implementation because the channel code and decoder can be designed independently from the modulation.

In fact, the coding and decoding could be done on the host processor rather than on the radio transceiver. All that would be required is that the host processor encode the message prior to passing it down to the transceiver, and that the transceiver pass the received codeword up to the host processor even if the message is received in error (e.g. error detection on the transceiver device must be turned off). If the decoding is performed on board the host processor, then so could the logic that controls the hybrid-ARQ protocol. Thus, it is conceivable that off-the-shelf Bluetooth or Zigbee devices could be used to implement a hybrid-ARQ protocol without any modification to the radio hardware. 
In this paper, we present an information theoretic analysis of the performance of hybrid-ARQ systems that are constrained to use noncoherent orthogonal modulation (which approximates the modulation used in Zigbee) and hard-decision decoding. The analysis follows that of [4] using the instantaneous capacity of hard-decision decoded noncoherent orthogonal modulation. This is the first contribution of this paper.

While the information-theoretic analysis assumes the use of capacity-approaching codes, such codes are generally not feasible in low-cost systems. Thus, the second contribution of this paper is to propose a coding technique suitable for hybridARQ with hard-decision decoding. The coding technique is based on the use of Reed-Solomon codes. The Reed-Solomon codeword is broken into several blocks, with five blocks being a convenient choice. We further propose using a selective combining decoding technique [6] that is appropriate for such a system. After the $B^{t h}$ block is received, a conventional hard-decision decoder will base its decision on all $B$ blocks. However, if one or more of the blocks has significantly more errors than the other, then the decoder will fail even if some blocks have only a few errors. In our selective codecombining technique which we call pseudo-soft, the decoder attempts several decoding passes, with each pass using one distinct combination of the $B$ received blocks that improves the chances of recovering the encoded message. The decoding technique is valid for Reed Solomon codes, since RS codes are typically able to detect the presence of uncorrectable errors.

As a final contribution, we contemplate the use of the proposed hybrid-ARQ technique in a relay network. The idea behind relaying is that if the network is dense enough, then it could be viewed as a large distributed antenna array. When a block is transmitted, it might be overheard by nodes other than the intended destination. If the destination requires an ARQ retransmission, the retransmission could come from one of theses nodes acting as a relay. Presumably if the relay is closer to the destination than the original source was, there will be some performance gain due to the reduced path loss when the second block is retransmitted. We proposed such a protocol in [7], where we termed it the HARBINGER (hybrid-ARQ based intra-cluster geographic relaying) protocol. However, as in [3] we assumed Gaussian input symbols. In the present paper, we revisit the HARBINGER protocol under the constraints of hard-decision decoded noncoherent orthogonal modulation.

\section{SYSTEM MODEL}

The system model is shown in Fig. 1. A message $\mathbf{u}$ of $k$ symbols is passed through a channel encoder to produce the codeword $\mathbf{c}$ of length $n$ symbols. Note that since we will later contemplate the use of Reed Solomon codes, the encoder does not need to be binary. The codeword is passed into a Mary orthogonal modulator. The $\mathrm{M}$-ary orthogonal modulator first transforms the codeword $\mathbf{c}$ into a sequence $\mathbf{d}$ of symbols $d_{i} \in\{0, \ldots, M-1\}$ through some mapping process. Each symbol $d_{i}$ in the sequence is used to select a symbol from the signal set $\mathcal{S}=\left\{s_{0}, \ldots, s_{M-1}\right\}$. Because the system uses orthogonal modulation, the symbol $s_{d_{i}}$ is the M-dimensional

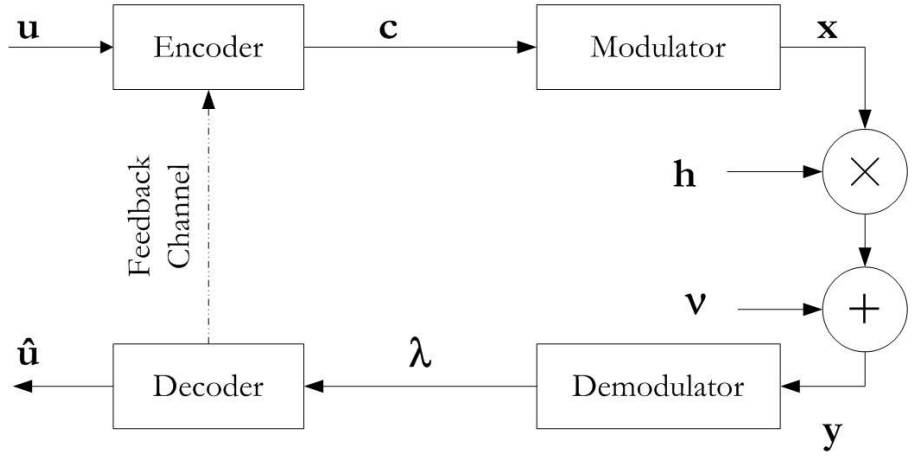

Fig. 1. A generic system model

elementary vector with a 1 in position $d_{i}$ and zeros elsewhere. The set of selected symbols are place into the $M$ by $N$ matrix $\mathrm{x}$ which are scaled such that the average energy per symbol $\mathcal{E}_{s}=E\left\{|x|^{2}\right\}$.

The matrix $\mathbf{x}$ is then broken into $B_{\max }$ equal length blocks, and blocks are sequentially transmitted until the receiver replies with a positive acknowledgement or all $B_{\max }$ blocks have been transmitted. Letting $\mathbf{x}[b]$ denote the $b^{\text {th }}$ block, $h[b]$ the complex fading coefficient for the block, and $\nu$ a vector of complex Gaussian noise whose dimensions match $\mathbf{x}[b]$ and whose components are zero-mean i.i.d. circularly symmetric Gaussian with variance $N_{o} / 2$ in each complex direction, the received signal during the $b^{\text {th }}$ block is:

$$
\mathbf{y}[b]=h[b] \mathbf{x}[b]+\nu .
$$

Note that because $h[b]$ is a scalar, it is assumed that the fading is constant for the entire duration of the block, and across all dimensions of the signal set. Without loss of generality, $E\left\{|h[b]|^{2}\right\}=1$ so that the average received energy per symbol is the same as the transmitted symbol energy.

Once a block has accumulated at the receiver, each received symbol in $\mathbf{y}[b]$ is passed through a demodulator that produces the log-likelihood ratio (LLR) estimates of the symbol. The demodulation is on a symbol-by-symbol basis and the demodulation process for a single symbol $y$ needs to be considered. For each possible $x_{m}, 1 \leq m \leq M$, a log-likelihood is formed:

$$
\begin{aligned}
\Lambda_{m} & =\log p\left(x_{m} \mid y\right) \\
& =\log \frac{p\left(x_{m} \mid y\right)}{\sum_{x \in \mathcal{S}} p(x \mid y)}
\end{aligned}
$$

where $p(x)$ is the pdf of $x$. For noncoherent orthogonal modulation without channel state information (CSI), the likelihood ratio given by [8] is:

$$
f\left(y \mid x_{m}\right)=\frac{\left(\frac{\mathcal{E}_{S}}{N_{o}}\right)^{2}\left|y_{m}\right|^{2}}{\frac{\mathcal{E}_{S}}{N_{o}}+1}
$$

Letting the likelihood $f(x \mid y)=\kappa p(x \mid y)$ for any arbitrary constant $\kappa$ that is common for all postulated symbols, and applying Bayes' rule, then (2) can be more conveniently 
rewritten as:

$$
\begin{aligned}
\Lambda_{m} & =\log \frac{f\left(y \mid x_{m}\right)}{\sum_{x \in \mathcal{S}} f(y \mid x)} \\
& =\log f\left(y \mid x_{m}\right)-\log \sum_{x \in \mathcal{S}} f(y \mid x) \\
& =\frac{\left(\frac{\mathcal{E}_{S}}{N_{o}}\right)^{2}\left|y_{m}\right|^{2}}{\frac{\mathcal{E}_{S}}{N_{o}}+1} .
\end{aligned}
$$

While the above is the optimal metric for soft-decision decoding, we further constrain our receiver to use hard-decision decoding. For hard-decision decoding, a symbol decision is made by picking the index of the largest $\Lambda_{m}$. It is this symbol that is passed to the decoder.

The decoding can proceed using the set of blocks $\{1, \ldots, B\}$ received up to time slot $B \leq B_{\max }$. The decoder is free to use all, one, or some subset of the received blocks. In the case that only the most recent block is passed through the decoder, the system is said to be a type-I hybrid-ARQ system. In typeII and type-III hybrid-ARQ, which are based on incremental redundancy, combinations of two or more accumulated blocks can be made. Because coding is done across the transmitted blocks (which essentially form a longer codeword for the same message), error correction performance can be more robust. The difference between the two is that the individual blocks in type-III hybrid-ARQ are also self-correctable whereas for type-II they are not.

\section{Discrete Memoryless Channel (DMC) CAPACITY}

The mutual information between channel input $X$ and output $Y$ is defined by [9] as:

$$
I(X, Y)=\sum_{y} \sum_{x} p(x, y) \log _{2} \frac{p(x, y)}{p(x) p(y)}
$$

where $p(x, y)$ is the joint pmf of $x$ and $y$ and $p(y)$ and $p(x)$ are the a priori probabilities of the occurrence of symbol $x$ or $y$ from $\mathcal{S}$. The capacity of a channel is found by maximizing the mutual information over all possible input distributions:

$$
C=\max _{p(x)} I(X, Y) \text {. }
$$

By assuming that $p(x)=1 / M$, the capacity in (6) is thus maximized. By using the fact that $p(y \mid x)=p(x, y) / p(x)$ and assuming that all outputs are equally likely (i.e $p(y)=1 / M$ for all symbols in $\mathcal{S}$ ), the mutual information in (5) becomes:

$$
\begin{aligned}
I(X, Y) & = \\
& =\sum_{x=1}^{M} p(y \mid x) \log _{2}(M p(y \mid x))
\end{aligned}
$$

We are using noncoherent $\mathrm{M}$-ary orthogonal modulation where the $P_{s}$ (probability of symbol error) is given by [10] as:

$$
P_{s}=\sum_{k=1}^{M-1}\left(\begin{array}{c}
M-1 \\
k
\end{array}\right) \frac{(-1)^{k+1}}{k+1} \exp \left[-\frac{k}{k+1} \frac{\mathcal{E}_{s}}{N_{0}}\right](9)
$$

where $M$ is the modulation order.

Once we calculate the $P_{s}$ for a particular signal to noise ratio, $\mathcal{E}_{s} / N_{o}$, it is straightforward to derive the symbol transition matrix. The probability of correctly receiving any symbol is $1-P_{s}$. The use of $M$ orthogonal basis functions implies that if a symbol error is made, any of the other $M-1$ symbols could have been sent with equal probability. Hence the probability that a specific symbol from $\mathcal{S}$ is received given that it is in error is simply $P_{s} /(M-1)$. In other modulation schemes such as QPSK or 16-QAM, the transition probability of an errant symbol would not have been equal for all symbols. Thus orthogonal signalling as in this case provides an easy way to compute the symbol transition matrix. The $M \times M$ symbol transition matrix, $T_{s}$, is:

$$
T_{s}=\left[\begin{array}{ccccc}
1-P_{s} & \frac{P_{s}}{M-1} & \cdot & \cdot & \frac{P_{s}}{M-1} \\
\frac{P_{s}}{M-1} & 1-P_{s} & \cdot & \cdot & \frac{P_{s}}{M-1} \\
\cdot & \cdot & \cdot & \cdot & \cdot \\
\cdot & \cdot & \cdot & \cdot & \cdot \\
\frac{P_{s}}{M-1} & \cdot & \cdot & \cdot & 1-P_{s}
\end{array}\right]
$$

$T_{s}(y, x)$ is $y^{t h}$ row and $x^{t h}$ column element. It is the conditional probability $p(y \mid x)$ that $y^{\text {th }}$ symbol was received given that $x^{t h}$ symbol was sent. Both $x$ and $y$ are any $\mathrm{M}$ possible symbols of signal constellation $\mathcal{S}$. Given $x=y$ then $T_{s}(y, x)=1-P_{s}$ which is the probability of successfully receiving the transmitted symbol $x$. Our mutual information equation (8) or equivalently the Discrete Memoryless Channel (DMC) capacity now becomes:

$$
\begin{aligned}
C= & \sum_{x=1}^{M} T_{s}(y, x) \log _{2}\left(M \times T_{s}(y, x)\right) \\
= & \log _{2} M+P_{s} \log _{2} \frac{P_{s}}{M-1} \\
& +\left(1-P_{s}\right) \log _{2}\left(1-P_{s}\right)
\end{aligned}
$$

\section{BLOCK FADING}

In block fading, the codeword is broken into $B$ blocks and each block is sent over an independent channel. The fading coefficient $h[b]$ of the $b^{\text {th }}$ block is constant for the entire duration of the block and the channel has a DMC capacity. Due to the fading coefficient, the instantaneous signal to noise ratio of the $b^{t h}$ block denoted by $\gamma_{b} \equiv|h[b]|^{2} \mathcal{E}_{s} / N_{0}$, alongside its corresponding capacity $C\left(\gamma_{b}\right)$ are also random. For Rayleigh block fading, $|h[b]|$ is Rayleigh and $|h[b]|^{2}$ is exponentially distributed.

Furthermore, when code-combining is used, then the combined capacity of the $B$ blocks is more than that of individual blocks, since each block is sent over an independent channel. For code-combining in soft decision channels, we add block capacities. However, for hard-decision DMC, we need a different approach. Whenever, the $B^{t h}$ block transmission is made, the cumulated codeword at the receiver has $B$ sets of blocks each with an independent $P_{s}$ due to varying instantaneous block signal to noise ratio $\gamma_{b}$. Suppose for $B=2$, the two transmitted blocks have symbol error probability $P_{s_{1}}$ and $P_{s_{2}}$. 
Thus the DMC has an error probability $P_{s_{1}}$ half the time and $P_{s_{2}}$ the other half.

For $B$ transmissions, the average probability of symbol error, $\bar{P} s$ becomes:

$$
\bar{P}_{s}=\frac{\left(P_{s_{1}}+P_{s_{2}}+. .+P_{s_{B}}\right)}{B}
$$

Using $\bar{P} s$ we can compute the accumulated DMC Capacity, $C\left(\gamma_{1}, \ldots, \gamma_{B}\right)$ using (10) and (11) respectively. The overall code-combined capacity is therefore:

$$
\begin{aligned}
C\left(\gamma_{1}, \ldots, \gamma_{B}\right)= & \log _{2} M+\bar{P}_{s} \log _{2} \frac{\bar{P}_{s}}{M-1} \\
& +\left(1-\bar{P}_{s}\right) \log _{2}\left(1-\bar{P}_{s}\right)
\end{aligned}
$$

When $B$ is finite, the channel is not ergodic, and therefore a Shannon-sense channel capacity does not exist. For finite $B$, a more relevant performance metric is the information outage probability, defined in [11] and [1] as the probability that the instantaneous capacity $C\left(\gamma_{1}, \ldots, \gamma_{B}\right)$ is less than the rate $R_{B}=$ $R / B$ :

$$
p_{0}(B)=P\left[C\left(\gamma_{1}, \ldots, \gamma_{B}\right)<R_{B}\right] .
$$

When $C\left(\gamma_{1}, \ldots, \gamma_{B}\right)$ is less than $R_{B}$, an information outage occurs. In block fading, the information outage probability is an information theoretic bound on the frame error rate (FER) of any system. In fig. (2), the FERs are derived by comparing the code rate against the channel capacity. Increase in $B$ (due to subsequent hybrid-ARQ retransmissions) causes this rate to fall relative to the DMC capacity and consequently the FERs improve. Since we have the same rate for both type-II and type-III hybrid-ARQ the theoretical results will be the same for both.

\section{Throughrut AnALYSis}

In order to provide the throughput analysis we borrow from the approach taken in [4]. Suppose the random variable $B$ indicates the number of hybrid-ARQ transmissions until a block is successfully received at the destination. In the case when $B$ is equal to an arbitrary value, $b$, the first $b-1$ attempts must have failed while the $b^{\text {th }}$ attempt has succeeded. The pmf of $B$ is thus:

$$
p_{B}[b]=\left(1-p_{0}(b)\right) \prod_{i=1}^{b-1} p_{0}(i) \text { for } b \geq 1
$$

Practical hybrid-ARQ protocols are limited to a maximum number of transmissions, which we denote as $B_{\max }$ If a message has not been correctly decoded after $B_{\max }$ transmissions, then the system moves on to the next message. The pmf of $B$, when constrained with $B_{\max }$ number of transmissions, is:

$$
p_{B}[b]= \begin{cases}\xi\left(1-p_{0}(b)\right) \prod_{i=1}^{b-1} p_{0}(i) & \text { for } 1 \leq b \leq B_{\max } \\ 0 & \text { otherwise }\end{cases}
$$

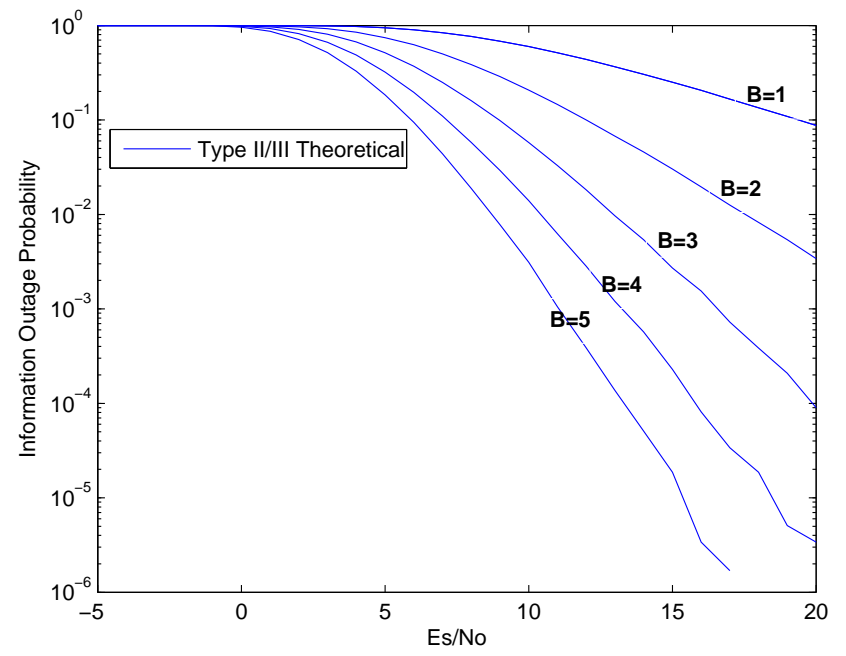

Fig. 2. Theoretical Frame Error Rates (FER) vs. SNR of noncoherent 16-ary orthogonal modulation. We assume a generic $(51,45)$ codeword for $\mathrm{B}=1$. By the fifth block transmission $(B=5)$, we equivalently have a $(255,45)$ codeword.

where $\xi$ is a normalization factor needed to make $p_{B}[b]$ a valid pmf:

$$
\xi=\left[\sum_{i=1}^{B_{\max }}\left(1-p_{0}(b)\right) \prod_{i=1}^{b-1} p_{0}(i)\right]^{-1} .
$$

Finally, we can derive the throughput efficiency, which is the ratio of correct bits to transmitted bits:

$$
\eta_{\text {eff }}=\frac{1-p_{0}\left(B_{\max }\right)}{E[B]} .
$$

where $E[B]$ is the expected value of transmissions needed for a given value of SNR. Some of the following figures showing comparison of throughputs are based on this metric.

\section{REEd SOLOMON CODING}

Reed Solomon (RS) codes are a class of non-binary block codes that have been extensively used for error correction in digital communication systems. An extensive review of the Reed Solomon codes is provided by [12]. A $(n, k)$ RS code operates over an alphabet of size $n+1$ and thus the number of bits per RS symbol is $\log _{2}(1+n)$. The number of correctible errors in a RS code is:

$$
t_{c}=\lfloor(n-k) / 2\rfloor
$$

We suppose a $(255,45)$ Reed Solomon mother codeword that is comprised of $45 \times \log _{2}(1+255)=360$ message bits that are encoded to $255 \times \log _{2}(1+255)=2040$ codeword bits. A 16-ary orthogonal modulation scheme implies that every $\log _{2}(16)$ or four bits from this codeword are transmitted as an orthogonal symbol. For every block, there are $2040 /(4 \times 5)=$ 102 orthogonal symbols transmitted over the channel.

The first Reed Solomon block is thus a $(51,45)$ codeword and is able to correct $(\lfloor(51-45)\rfloor / 2=3$ errors. If due to fading and low signal to noise ratio, the receiver is unable 
to decode the codeword, the next block from the original codeword is transmitted over the channel. At the receiver, the new block is code-combined with the original block to yield an error correcting performance of $(\lfloor(51 \times 2-45)\rfloor / 2=28$ symbols for the two blocks. This process of block transmissions continues until the destination is able to decode the original message or the maximum number of transmissions are made. This incremental redundancy allows a larger number of errors to be corrected for successive block transmissions. Using (19), the correctable errors capability for the 5 transmissions of a $(255,45)$ mother code are $3,28,54,79$ and 105 errors respectively.

The drawback of the type-II approach is that if there are a very large number of errors for one block, there is a chance that if another block transmission is made, the total number of accumulated errors would still be too large for error correction capability of the combined blocks. Putting it in another way, it is possible that individual blocks or combinations of blocks are correctable even though the thus far accumulated codeword may not be. This is in contrast to the information theoretic approach where the channel capacity can only increase with incremental redundancy.

In [6], a combinatoric code combining technique for RCPC (Rate-compatible Punctured Convolutional) codes was presented. In this hybrid-ARQ approach, the receiver makes selective combinations of the blocks to maximize the chances of successfully decoding the message. We can use this idea for Reed Solomon codes to exploit the incremental redundancy in a better way than the type-II hybrid-ARQ. Suppose in four transmissions of $(51,45)$ blocks from the original mother code of $(255,45)$, the block errors are 15, 51, 21 and 17. The total accumulated symbol errors are 104, which is beyond the limit of 79 correctable errors of four transmissions. However a closer look would reveal that blocks 1, 3 and 4 have 53 errors in total which is within the limit of 54 correctable errors for three blocks.

In our pseudo-soft approach, encoding is done in such a way that for every transmission $B$, the receiver looks to see if individual blocks or combinations of blocks can be decoded. If the hard-decision decoding fails, the system will have to make up to $\sum_{i=1}^{B_{\max }}\left(\begin{array}{c}B_{\max } \\ i\end{array}\right)$ combinations of received blocks and compare against correctable errors for transmission $B$ to see if the message can be decoded. The pseudo-soft typeIII hybrid-ARQ provides significant dividends because due to block fading the instantaneous signal to noise ratio of a combination of random blocks might be enough for the message to be decoded even though the codeword on the whole might be in outage. The gain is shown in fig. (3).

For the sake of comparison, we have also examined the throughput performance of type-I hybrid-ARQ as shown in fig. (4). Upon the failure of the receiver in decoding the symbol errors of a block, a retransmission is made. The receiver then tries to decode the new block without combining it with the previous transmission.

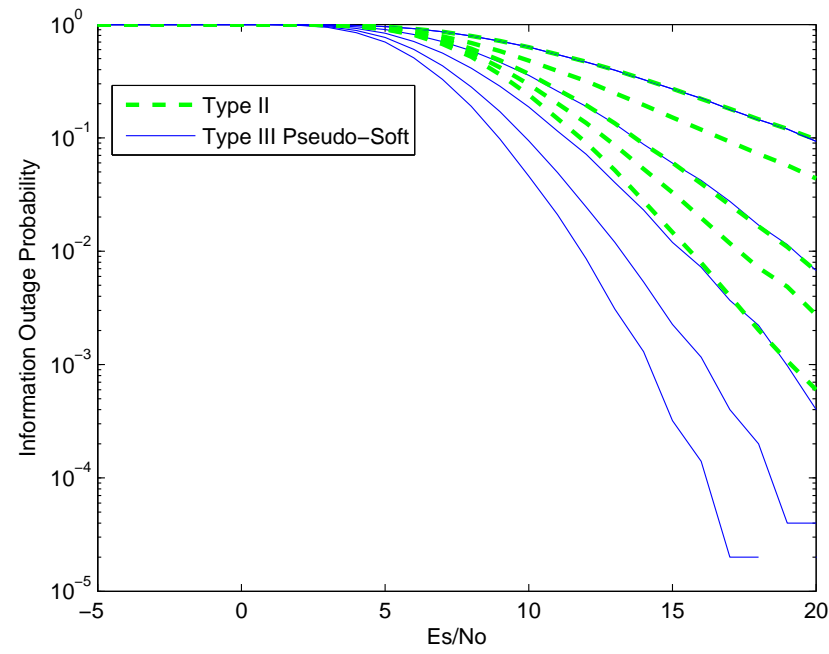

Fig. 3. Frame Error Rates (FER) vs. SNR for noncoherent 16-ary orthogonal modulated hybrid-ARQ with 5 block transmissions $\mathrm{B}=1,2, ., 5$ of a $(255,45)$ Reed Solomon mother code.

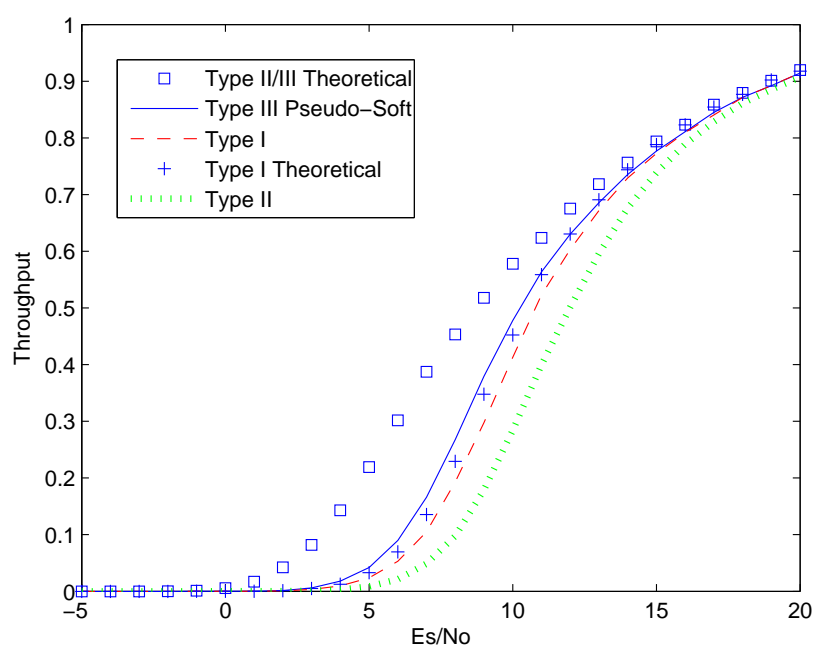

Fig. 4. The throughput efficiency curves of noncoherent 16-ary orthogonal modulated hybrid-ARQ vs. SNR with a $(255,45)$ Reed Solomon mother code. The Theoretical curves represent the computation of throughput by comparing the DMC capacity against the code rate rather than by using the actual code.

\section{NETWORK RELAYING}

In a relaying network, relay nodes help the transmission of a message from the source to destination particularly at low signal to noise ratios. In [7] the HARBINGER (hybridARQ based intra-cluster geographic relaying) protocol was presented. Although the initial hybrid-ARQ block comes from the source, subsequent block transmissions may come from any relay that has successfully decoded the message and is closest to the intended destination. Hence the time-diversity benefit of hybrid-ARQ is further enhanced by the spatialdiversity effect of relaying. This is in contrast to conventional hybrid-ARQ where retransmitted packets also have to come 


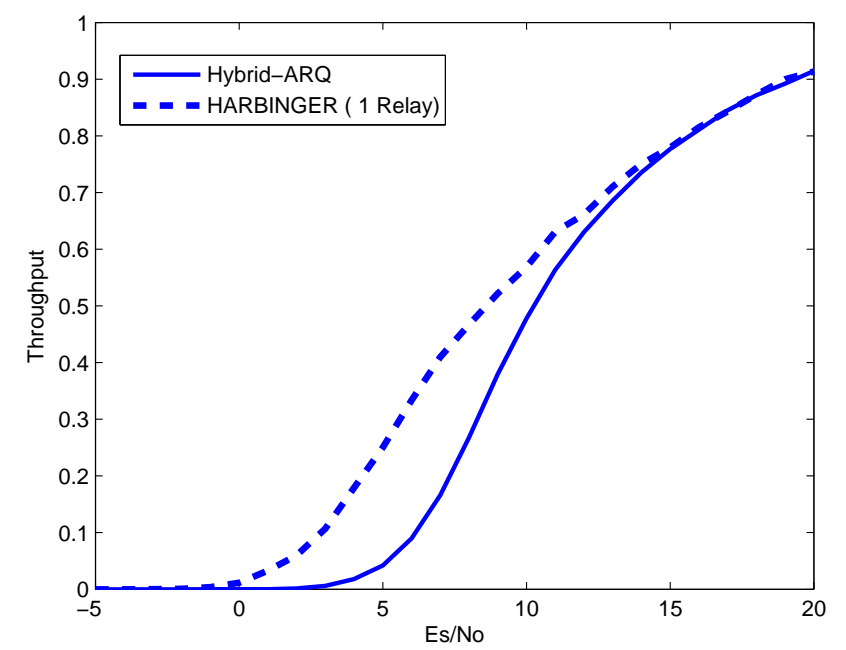

Fig. 5. The throughput efficiency vs SNR in a comparison of point-to-point and relay-based transmission. The results are derived for the Type III pseudosoft technique using a $(255,45)$ mother code .

from the original source. The performance of HARBINGER in [7] demonstrated a significant improvement in the energylatency tradeoff when compared with conventional multihop protocols. In fig.(5), a relay positioned midway between the source and destination in an environment with a path loss coefficient of 2 is assumed. A significant improvement in throughput efficiency at low signal to noise ratios can be observed.

In [13], Turbo codes were implemented for the Bluetooth standard by encoding messages for the AUX1 packets, without requiring any modification to the existing standard. The results of [13] showed significant improvement in throughput and reduction in latency at low signal to noise ratios. Likewise, the HARBINGER protocol using the pseudo-soft coding can be incorporated into the applications layer of a broadcast-oriented network based on the IEEE 802.15.4 standard. The Zigbee protocol for the $2450 \mathrm{MHZ}$ band does not use truly orthogonal modulation, but rather Walsh codes with O-QPSK using 16-ary symbols that are nearly orthogonal [5]. Therefore our results for noncoherent 16-ary orthogonal modulation should also be approximated for the Zigbee standard.

The Zigbee standard uses node clusters communicating either on a star or a peer-to-peer topology. Each cluster is assigned an ID number and is controlled by a coordinator node alongside CSMA-CA mechanism for medium access control [5]. The HARBINGER protocol lends itself to a peer-to-peer topology since intra-cluster communication does not need to be routed through the coordinator. This is in contrast to the star topology where all messages are communicated through the coordinator [5]. In the applications layer, the message can be encoded and then transferred to the lower layers as payload to be transmitted as as part of the Data Frame. In case of failure in decoding the message, subsequent Data Frames would carry the payloads corresponding to the remaining blocks of the encoded message.

\section{CONCLUSIONS}

The throughput of a practical hybrid-ARQ system in block fading suffers loss relative to the information theoretic bound because of modulation and coding constraints. We have looked at both these constraints by using noncoherent 16-ary orthogonal modulation and a Reed Solomon code. Additionally, our paper has looked at theoretic performance limits of hybridARQ based on a hard-decision DMC capacity. Prior work had either assumed unconstrained modulation or soft-decision capacities. To reiterate, the DMC capacity is a more appropriate comparison parameter for low-power hard-decision digital radios. A selective code-combining technique has been used that maximizes the decoding capability of a mother code. We have suggested a basic framework for running a decodeand-forward protocol like HARBINGER for the Zigbee-based networks. As for the coding scheme, future work could also consider RCPT (rate compatible punctured Turbo) codes [14] or the rate-less raptor codes [15].

\section{ACKNOWLEDGMENTS}

This work was supported by Augusta Systems in support of phase-II Navy STTR contract number N68335-05-C-0303.

\section{REFERENCES}

[1] L. Ozarow, S. Shamai, and A. D. Wyner, "Information theoretic considerations for cellular mobile radio," IEEE Trans. Veh. Tech., vol. 43, pp. 359-378, May 1994.

[2] S. Wicker, Error Control Systems for Digital Communications and Storage. Englewood Cliffs, NJ: Prentice Hall, Inc., 1995.

[3] G. Caire and D. Tuninetti, "The throughput of hybrid-ARQ protocols for the Gaussian collision channel," IEEE Trans. Inform. Theory, vol. 47, no. 5, pp. 1971-1988, July 2001.

[4] T. Ghanim and M. C. Valenti, "Throughput of hybrid-arq under modulation constraints for block fading," in Conf. on Inform. Sciences and Systems (CISS), Princeton, NJ, Mar. 2006.

[5] LAN/MAN Standards Committee of the IEEE Computer Society, "Draft standard for part 15.4: Wireless medium access control (MAC) and physical layer (PHY) specifications for low rate wireless personal area networks (LR-WPANs)," Draft P802.15.4/D18, pp. 27-52, Feb. 2003.

[6] Q. Zhang and S. Kassam, "Hybrid ARQ with selective combining for video transmission over wireless channels," in International Conference on Image Processing, Santa Barbara, CA, Oct. 1997.

[7] B. Zhao and M. C. Valenti, "Practical relay networks: A generalization of hybrid-ARQ," JSAC, vol. 1, Jan. 2005.

[8] S. Cheng and M. C. Valenti, "Iterative demodulating and decoding of turbo-coded m-ary noncoherent orthogonal modulation," IEEE J. Select. Areas Commun., vol. 23, no. 9, Sep. 2005.

[9] T. M. Cover and J. A. Thomas, Elements of Information Theory. Wiley, 1991.

[10] R. E. Ziemer and R. L. Peterson, Introduction to Digital Communication, 2nd ed. Pearson Education, 2004.

[11] R. Knopp and P. A. Humblet, "On coding for block fading channels," IEEE Trans. Inform. Theory, vol. 46, no. 1, pp. 189-205, Jan. 2000.

[12] S. Lin and D. Costello, Error Control Coding, 2nd ed. Upper Saddle River, NJ: Prentice Hall, 2004.

[13] M. C. Valenti and M. Robert, "Improving the qos of bluetooth through turbo coding," in MILCOM, Los Angeles, CA, 2002, pp. 1057-1061.

[14] D. N. Rowitch and L. B. Milstein, "On the performance of hybrid FEC/ARQ systems using rate compatable punctured turbo (RCPT) codes," IEEE Trans. Commun., vol. 48, no. 6, pp. 948-959, June 2000.

[15] A. Shokrollahi, "Raptor codes," in Proc. IEEE Int. Symp. on Inform. Theory (ISIT), Chicago, July 2004. 